[Review]

\title{
单一导电属性及手性单壁碳纳米管的分离技术
}

\author{
李红波张 静 金赫华 李清文* \\ (中国科学院苏州纳米技术与纳米仿生研究所, 江苏苏州 215123)
}

\begin{abstract}
摘要：综述了基于生长后处理策略下单一导电属性及手性单壁碳纳米管(SWCNTs)分离技术的研究进展, 阐 述了 SWCNTs 的选择性分离原理, 并比较了不同分离技术在分离纯度、效率、成本以及可规模化等方面的优缺 点, 为 SWCNTS 分离技术的发展及应用提供了方向性指导.
\end{abstract}

关键词：单壁碳纳米管；分离技术；导电属性；手性

中图分类号: 0649

\section{Separation Techniques of Single-Walled Carbon Nanotubes with Single Electrical Type and Chirality}

\author{
LI Hong-Bo Z ZHANG Jing JIN He-Hua LI Qing-Wen* \\ (Institute of Nano-tech and Nano-bionics, Chinese Academy of Sciences, Suzhou 215123, Jiangsu Province, P. R. China)
}

\begin{abstract}
Based on the solution post-synthesis method, we reviewed the process in the separation techniques of single-walled carbon nanotubes (SWCNTs) with single electrical type and chirality. We demonstrated the separation mechanism of SWCNTs by the different methods and comparatively pointed out their merits and disadvantages in purity, efficiency, cost, and scalability etc. Furthermore, some prospects for future study and application were proposed.
\end{abstract}

Key Words: Single-walled carbon nanotube; Separation technique; Electrical type; Chirality

1 引言

单壁碳纳米管(SWCNTs) 可看作是由单层石墨 绕中心轴按一定的螺旋角卷曲而成的无缝空心管. 不同的卷曲角度和曲率决定了 SWCNTs 的不同性 质. SWCNTs 的直径、螺旋型等结构特征由卷曲矢量 (Ch) 唯一确定, 手性矢量可写成 $\mathrm{Ch}=n \boldsymbol{a}_{1}+m \boldsymbol{a}_{2} \equiv(n, m)$ 的形式. 其中, $\boldsymbol{a}_{1}$ 和 $\boldsymbol{a}_{2}$ 为石墨层六角网格的基矢, $n$, $m$ 均为整数, $(n, m)$ 与 SWCNTs 的导电性能密切相 关, 对于一个给定 $(n, m)$ 的纳米管, 如果 $2 n+m=3 q(q$ 为整数), 则 SWCNTs 是良好的导体, 表现出金属特 性, 否则表现为半导体特性. 金属型(m-)SWCNTs 具
有优良的导电性能, 其导电率可以比铜高 2-3 数量 级, 在高导电性的透明电极或集成电路用纳米级金 属导线、太阳能电池、触摸屏、电子纸等方面有望得 到应用: ${ }^{1,2}$ 而半导体型(s-)SWCNTs 具有优异的电子 迁移率, 在高灵敏度化学与生物传感器、高性能纳 电子器件、场效应晶体管(FETs)及纳米集成电路制 作等领域有着良好的应用前景. ${ }^{4-6}$ 但是, 目前通过常 规制备手段如激光蒸发法、电弧放电法与化学气相 沉积法等所制备的 SWCNTs 均是不同导电属性和 手性的 SWCNTs 混合物, 极大地阻碍了其特异电子 性能的发挥及在诸多高端科技领域里的潜在应用.

Received: July 4, 2012; Revised: September 4, 2012; Published on Web: September 4, 2012.

*Corresponding author. Email: qwli2007@sinano.ac.cn; Tel: +86-512-62872577.

The project was supported by the National Key Basic Research Program of China (973) (2011CB932600) and National Natural Science Foundation of China (20903069, 21073223).

国家重点基础研究发展规划项目(973)(2011CB932600)及国家自然科学基金(20903069,21073223)资助

C. Editorial office of Acta Physico-Chimica Sinica 
因此, 如何有效地获得具有单一导电属性及手性 SWCNTs 是实现其在电子及光电器件等领域的材料 基础和应用前提.

目前, 为了获得单一导电属性或手性的 SWCNTs, 研究者们主要采取以下三个策略: (1) 直 接生长; (2) 选择性消除法; (3) 选择性分离技术. 2004 年斯坦福大学 Dai 研究组 ${ }^{7}$ 最先利用等离子体 化学气相沉积(PECVD) 设备在低温下直接生长获得 了 $90 \%$ 纯度的 s-SWCNTs. 随后戴顿大学 Dai 研究 组 ${ }^{8}$ 通过快速生长获得了 $96 \%$ 纯度的 s-SWCNTs, 并 直接用于制备 FETs 器件. 此外, 研究者们利用普通 化学气相沉积(CVD)设备通过控制碳源或者生长过 程中原位地引入紫外光束也可以有效地抑制 m-SWCNTs 的生长, 从而得到 s-SWCNTs. ${ }^{9}$ ' 10 国科 学院物理研究所 Bai 研究组 ${ }^{11}$ 则在化学气相沉积过 程中将 $\mathrm{B} / \mathrm{N}$ 掺杂到 SWCNTs 上, 将 $\mathrm{m}-\mathrm{SWCNTs}$ 转变 为 s-SWCNTs, 直接用于制备 FETs 器件. 2009 年北 京大学 Zhang ${ }^{12}$ 和 $\mathrm{Liu}^{13}$ 研究组利用克隆技术以开口 的 SWCNTs 或开口的富勒烯 $\left(\mathrm{C}_{60}\right)$ 为催化剂种子复制 SWCNTs, 在直径和手性控制方面取得了重要进 展. 消除法则是另一种被较早使用获得 $\mathrm{m}-\mathrm{SWCNTs}$ 或 s-SWCNTs 的策略. 利用大电流烧蚀、 ${ }^{14}$ 离子刻蚀、 ${ }^{15}$ 激光照射 ${ }^{16,17}$ 等物理手段可有效消除 m-SWCNTs, 得 到 s-SWCNTs. 使用 $\mathrm{SO}_{2}$ 气体 ${ }^{18}$ 则可选择性刻蚀 s-SWCNTs, 而 m-SWCNTs 被保留. 通过消除法分离 的 SWCNTs, 尤其是高密度、水平 s-SWCNTs 阵列, 可以直接用于纳米电子器件, 从而避免了生长后分 离过程中杂质的引入, 在高开关比FETs 及传感器件 上都将有很好的应用前景. 但是, 基于这两种方法 所制备的 SWCNTs 在规模化和手性控制方面还存 在很大的困难.

相比于直接生长和选择性消除法, 基于生长后 处理的选择性分离技术近年来得到了长足的发展. 主要的选择性分离技术包括: 选择性化学修饰、交 流介电泳、梯度密度离心法、脱氧核糖核酸(DNA)包 裹的离子交换色谱法, 凝胶电泳及柱色谱法等. 研 究者不仅实现了金属/半导体型 $(\mathrm{m} / \mathrm{s}) \mathrm{SWCNTs}$ 分离, 一些分离技术也实现了单一手性 SWCNTs 的分离. 本文从 SWCNTs 表面性质、直径以及手性结构出 发, 在对 SWCNTs 的 $\mathrm{m} / \mathrm{s}$ 及手性分离技术进行综述 的同时, 也着重讨论了不同分离技术下SWCNTs 的 $\mathrm{m} / \mathrm{s}$ 及手性分离原理, 从而为研究者们提供方向性 指导和参考.

\section{2 单壁碳纳米管分离技术}

\section{1 选择性化学修饰}

理论计算和实验结果表明, SWCNTs 的电子结 构、直径和手性可以影响其与一些化学物质如有机 小分子、表面活性剂、DNA、共轭聚合物等的相互 作用力. 利用这种相互作用力, 研究者们可以对 SWCNTs 进行选择性共价或非共价修饰, 从而通过 简单的离心和过滤实现分离.

与 s-SWCNTs 相比, m-SWCNTs 在费米能级处 具有较高的电子态密度和较小的电离电势, 这导 致 m-SWCNTs 比 s-SWCNTs 具有更高的反应活 性. 利用此差异, 对 SWCNTs 的侧壁进行共价修 饰, m-SWCNTs 则被选择性地溶解在溶液中, 而 s-SWCNTs 则留在固体相中. 2003 年 Strano 和 Tour ${ }^{19}$ 研究组合作报道了控制反应条件重氮试剂可以选 择性地与 m-SWCNTs 反应, 使其溶解在水溶液中与 $\mathrm{s}-\mathrm{SWCNTs}$ 分离. 后来, Weisman 和 Tour 合作研究组 ${ }^{20}$ 发现重氮盐对于 $m$-和 s-SWCNTs 均具有反应活性, 只是在反应速率上表现出差异, 使用苯基取代的重 氮盐则对具有最大能级带隙的 s-SWCNTs 展现了最 高的反应活性. 硝鎓离子 ${ }^{21}$ 或 $\mathrm{OsO}_{4}{ }^{22}$ 也可以选择性 修饰 m-SWCNTs, 而甲亚胺内鎓盐则可通过环化 加成对 s-SWCNTs 进行选择性修饰. ${ }^{23}$ 此外 Kamaras 研究组 ${ }^{24}$ 使用二氯卡宾利用共价化学方法将 $\mathrm{m}$ SWCNTs 转化为 s-SWCNTs. 但是, 共价修饰对于 SWCNTs 的反应选择性不易控制并且表面结构的破 坏限制了其发展和应用。

近年来 SWCNTs 的非共价修饰备受青睐. 2003 年 Papadimitrakopoulos 研究组 ${ }^{25}$ 报道了十八烷基胺 (ODA) 对羧基化的 s-SWCNTs 具有较强的吸附, 可 以选择性地将其溶解在溶液中, 实现与 m-SWCNTs 的分离. 类似地, 溴 ${ }^{26}$ 和卟啉 ${ }^{27}$ 也可以对 $\mathrm{s}-\mathrm{SWCNTs}$ 具有选择性修饰特性, 将其选择分散在溶液中, 而 m-SWCNTs 则通过离心沉淀出来. 进一步地设计分 散剂结构, 研究者们还可以实现 SWCNTs 的直径及 手性分离. Papadimitrakopoulos 研究组 ${ }^{28}$ 发现黄素单 核苷酸分子在碳管表面上可以通过氢键相互作用 形成螺旋状组装体, 其结构强烈依赖于 SWCNTs 的 手性结构, 从而选择性地分散 $(8,6)$ 碳管. Rahman 和 Komatsu 研究组 ${ }^{29}$ 则使用手性的双叶啉 “纳米铝子” 实现了对 $(6,5)$ 碳管的富集. 除了小分子化合物, 一 些芳香型的共轭聚合物也可以实现 SWCNTs 的 $\mathrm{m} / \mathrm{s}$ 及手性分离. 2007 年 Nicholas 研究组 ${ }^{30}$ 首先报道了 
聚芴类高分子可以选择性地分散 s-SWCNTs, 并且 不同结构的聚合物表现出不同的选择性. 2011 年 Kim 和 Bao 研究组 ${ }^{31}$ 合作报道了结构规整的 3-烷基 聚噻吩也可以选择性分散 s-SWCNTs, 所制备的 FET 器件的开关比高达 $10^{6}$ 以上. 进一步地改变芳香 类共轭聚合物结构, 其它共轭聚合物也可以实现对 S-SWCNTs 的选择性分散, 并表现出一定的直径选 择性. ${ }^{32-35}$ 由于共轭聚合物自身的电学特性, 所制备 的 SWCNT 复合物可直接用于光伏和 FETs 等器件 上, 并且对 s-SWCNTs 的掺杂显著地提高了其器件 性能. 选择性化学修饰法给予了化学家们丰富的想 象和探索空间, 也预示着更多的功能分子可以应用 于 SWCNTs 的选择性分离. 尽管此法在 SWCNTs 的宏量分离上具有一定的优势, 但是在更多手性 SWCNTs 的分离方面依然面临巨大的挑战.

\section{2 交流介电泳法}

在电场作用下, SWCNTs 通常会被诱导并表现 出一定的偶极矩, 选择合适的不均匀的电场就可 以使悬浮的 SWCNTs 以特定方式排列和运动. 由 于 $\mathrm{m}-\mathrm{SWCNTs}$ 和 s-SWCNTs 分别是弹道导体和半 导体, 理论计算结果表明, 在交流电场作用下 m-SWCNTs 和 s-SWCNTs 在溶剂中会展现出不同的 介电常数, 从而进行反向运动. 利用这种差异, 2003 年 Krupke 和 Hennrich 研究组 ${ }^{36}$ 共同发展了基于交 流介电泳法的 SWCNTs 的 $\mathrm{m} / \mathrm{s}$ 分离技术. 在微电 极阵列上, 使用 $10 \mathrm{MHz}$ 的频率和 $10 \mathrm{~V}$ 的峰电压, $\mathrm{m}$-SWCNTs 和 s-SWCNTs 在电场中进行反向运动, 在悬浮液中的 m-SWCNTs 被微电极阵列吸引发生 沉积, 而 s-SWCNTs 保留在溶液中实现 $\mathrm{m} / \mathrm{s}$ 分离, 如 图 1(a)所示. 此后, 研究者们相继对该技术进行了改 进, 在分离纯度、SWCNTs 有序排列以及小量分离上
取得了一定的进展. 例如使用 $\mathrm{H}$-形的微流体通道, 可以持续地富集 $\mathrm{m}-\mathrm{SWCNTs}$ 和 s-SWCNTs 的溶液, ${ }^{37}$ 如图 1(b)所示. 但是, 受到微电极阵列的限制, 该方 法在分离纯度、分离效率等方面很难得到实质性提 高, 限制了其发展和进一步的应用.

\section{3 梯度密度超高速离心法}

梯度密度超高速离心法(DGU)是在一种广泛用 于生物化学以及医药学上的亚细胞、DNA 和核糖核 酸(RNA)的分离技术. 2005 年 Hersam 研究组 ${ }^{38}$ 率先 将 DGU 技术应用于 DNA 包裹 SWCNTs 的直径分 离. 随后, 他们报道了可以使用胆酸钠(SC)表面活 性剂分散的 SWCNTs, 并根据碳管的直径、带隙、 电子类型进行分离. ${ }^{39}$ 其分离原理是利用单分散的 SWCNTs 的浮游密度与其几何结构和表面活性剂涂 层厚度的相关性, 将 SWCNTs 分散液注入到具有线 性密度梯度的碘克沙醇水溶液中离心, 在离心力的 作用下, 不同管径的 SWCNTs 在不同密度梯度环境 中逐渐接近各自的平衡密度, 从而实现分离, 如图 2 (a)所示. 在此基础上, 他们发现除了 DNA 分子和表 面活性剂, 使用非离子、生物相容性的聚丙二醇与 环氧乙烷的嵌段共聚物(pluronics) 分散 SWCNTs 也 可以实现 SWCNTs 的 $\mathrm{m} / \mathrm{s}$ 分离. ${ }^{40}$ 较短疏水链的 Pluronic 可以实现 $99 \%$ 以上纯度 s-SWCNTs 的富集, 相 反 $\mathrm{X}$ 形的四季醇共聚物则有利于 $\mathrm{m}-\mathrm{SWCNTs}$ 的富 集, 纯度可以达到 $74 \%$.

此法除了适用于不同导电属性的 SWCNTs 分 离, 更细致地调控 SWCNTs 表面包裹结构及分离条 件外, 还可以实现 SWCNTs 的手性甚至镜像异构体 的分离. 2009 年 Hersam 研究组 ${ }^{41}$ 进一步使用手性结 构的 SC 表面活性剂分别获得了左手和右手螺旋的 SWCNTs. 而 Maruyama 研究组 ${ }^{42}$ 使用脱氧胆酸钠
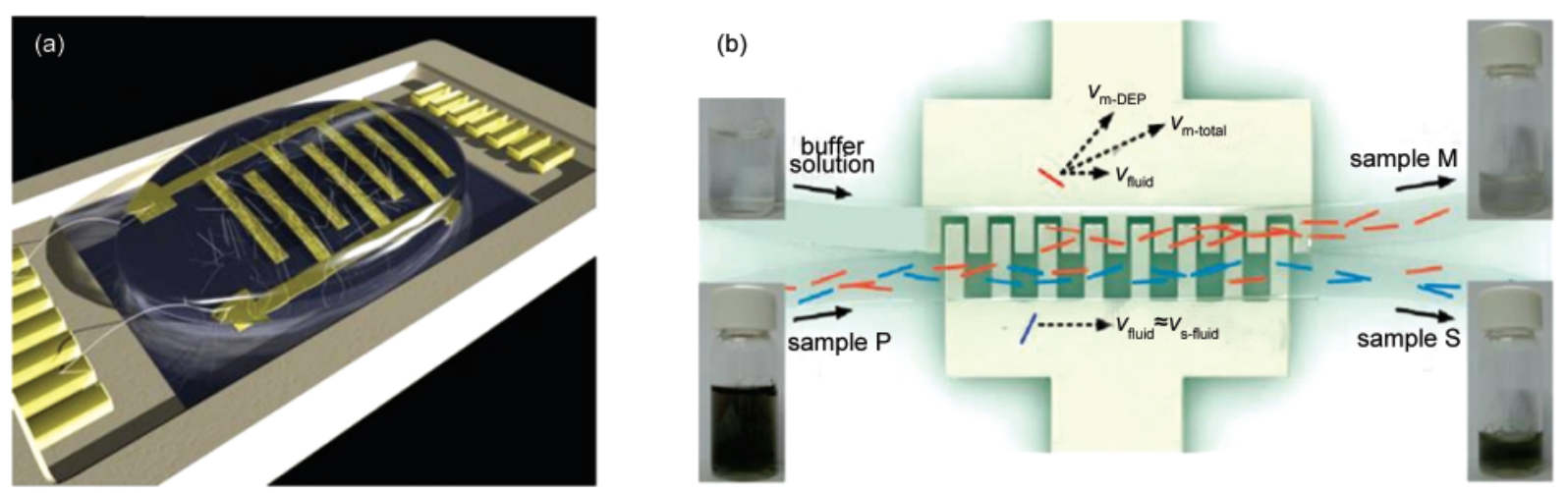

图 1 (a) SWCNT 悬浮液在微电极阵列上的交流介电泳示意图 ${ }^{36}$ 和(b)使用 H-形的微流体通道的交流介电泳连续分离机理 ${ }^{37}$

Fig.1 (a) Schematic of dielectrophoresis of SWCNT suspension by using a microelectrode array ${ }^{36}$ and (b) the continuous separation mechanism based on dielectrophoresis by using an $\mathrm{H}$-shaped microfluidic channel ${ }^{37}$ 
(a)

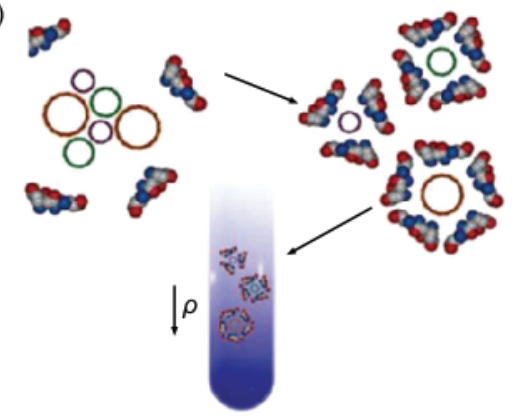

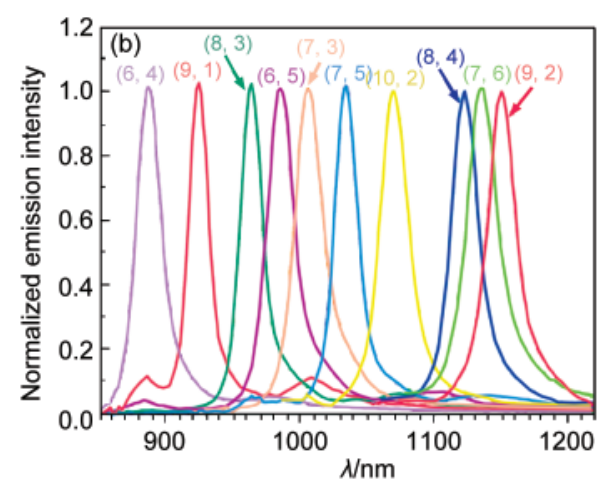

图 2 (a) 表面活性剂在 SWCNTs 上的包裹示意图 ${ }^{39}$ 和(b) 利用非线性梯度密度离心法获得的 10 种 $(n, m)$ 手性的 s-SWCNTs 的荧光光谱谱图 ${ }^{43}$

Fig.2 (a) Schematic of surfactant encapsulation on SWCNTs ${ }^{39}$ and (b) photoluminescence spectra of 10 kinds of separated $(n, m)$ s-SWCNTs using nonlinear DGU ${ }^{43}$

(DOC)与十二烷基硫酸钠(SDS)共表面活性剂分散 SWCNTs, 通过 DOC 对 SDS 在 SWCNTs 表面包裹结 构的调制获得了 $97 \%$ 纯度的 $(6,5)$ s-SWCNTs. 但是, 利用此法在一个分离程序下同时富集不同结构的 SWCNTs 面临很大的困难. 2010年 Weisman 研究组 ${ }^{43}$ 在此方面获得了重大突破, 通过非线性密度梯度 分离程序同时获得了 10 种不同手性的 s-SWCNTs, 如图 2(b) 所示, 进一步调制分离过程还实现了几种 手性碳管的镜像异构体的分离.

总之, 通过梯度密度超高速离心法, 研究者已 经可以富集直径差异 $0.02 \mathrm{~nm}$ 的 SWCNTs, 分离纯 度甚至可以高达 $99 \%$, 在 SWCNTs 的高纯度及手性 富集方面具有明显优势, 但是也存在难以大规模分 离, 设备要求高、耗时、取样操作难等问题.

\section{4 离子交换色谱法}

离子交换色谱主要用于带电离子以及极性物 质如多肽、蛋白质、核苷酸、核苷和各种碱基的分 离. 使用 DNA 来包裹 SWCNTs 时, SWCNTs 就可以 静电吸附在带有正电荷的阴离子交换树脂中. 不同 直径和电子特性 SWCNTs 的表面表现出不同的静 电学性质, 会导致 SWCNTs 与阴离子交换树脂间亲 和力的差异, 从而使用不同离子强度的带相反电荷 的流动相可以将结合在离子交换树脂上的 SWCNTs 分别交换下来, 实现分离.

2003 年 Zheng 研究组 ${ }^{44,45}$ 最早发现了通过 $\pi$ 相 互作用单链的 DNA 可以螺旋缠绕于 SWCNTs 表 面, 有效地分散 SWCNTs, 并且使用合适的离子交换 色谱获得了 SWCNTs 的 $\mathrm{m} / \mathrm{s}$ 分离. 对 $\mathrm{m}-\mathrm{SWCNTs}$ 来 说, DNA 上离散的负电荷形成一个沿轴向的静电 场, 在碳管中感应出正的净电荷, 复合物的净线电
荷密度与 DNA 分子相比就减少了. 对 s-SWCNTs 来 说, 碳管具有更低的极化率, DNA-SWCNT 复合物 的有效线电荷密度与 DNA 分子相比增大了. 因此, $\mathrm{m}$-SWCNTs 和 s-SWCNTs 与阴离子交换树脂间的静 电吸附强度表现出很大差异, m-SWCNTs 会被优先 洗脱下来, 实现了与 $\mathrm{s}-\mathrm{SWCNTs}$ 的分离.

但是, DNA 分散的 SWCNTs 具有较宽的长度 分布范围, 这在一定程度上影响了分离分辨度. 因而, 2007 年他们 ${ }^{46}$ 首先使用尺寸排阻色谱对 SWCNTs 进行了长度分离, 获得了窄长度分布的碳 管, 再使用离子交换色谱获得了 $(9,1)$ 和 $(6,5)$ 手性 碳管. Dai 研究组 ${ }^{47}$ 随后也报道了利用 DNA 在不 同管径和螺旋度型 SWCNTs 上包裹所形成复合 物的电荷密度差异, 通过离子交换色谱实现了 $\mathrm{m}$ SWCNTs 和窄手性分布 s-SWCNT 的分离, 其中由 小直径 s-SWCNTs 制作的 FET 器件的开关比高达 $10^{5} .2009$ 年 Zheng 研究组 ${ }^{48}$ 使用了一系列的单链 DNA 序列来包裹 SWCNTs, 在 SWCNTs 手性分离上 获得重大突破, 他们成功地实现了 12 种单一手性 SWCNTs 的分离, 如图 3(a)所示. 通过研究 20多种短 链的 DNA 序列, 他们认为 DNA 分子中的嘌呤和嘧 啶通过氢键相互作用形成周期性二维的薄层(图 3(b)), 进而选择性地包裹碳管形成桶状结构, 如图 $3(\mathrm{c})$ 所示. 此分离技术的优点是通过设计不同结 构单链 DNA 来包裹 SWCNTs 可以极大地提高对 SWCNTs 的表面性质的识别和分辨度, 但是这种特 定结构的单链DNA 分子成本极高, 限制了此方法的 广泛应用。

\section{5 基于糖基凝胶介质的分离技术}

SWCNTs 是典型的一维纳米材料, 直径在 0.3- 

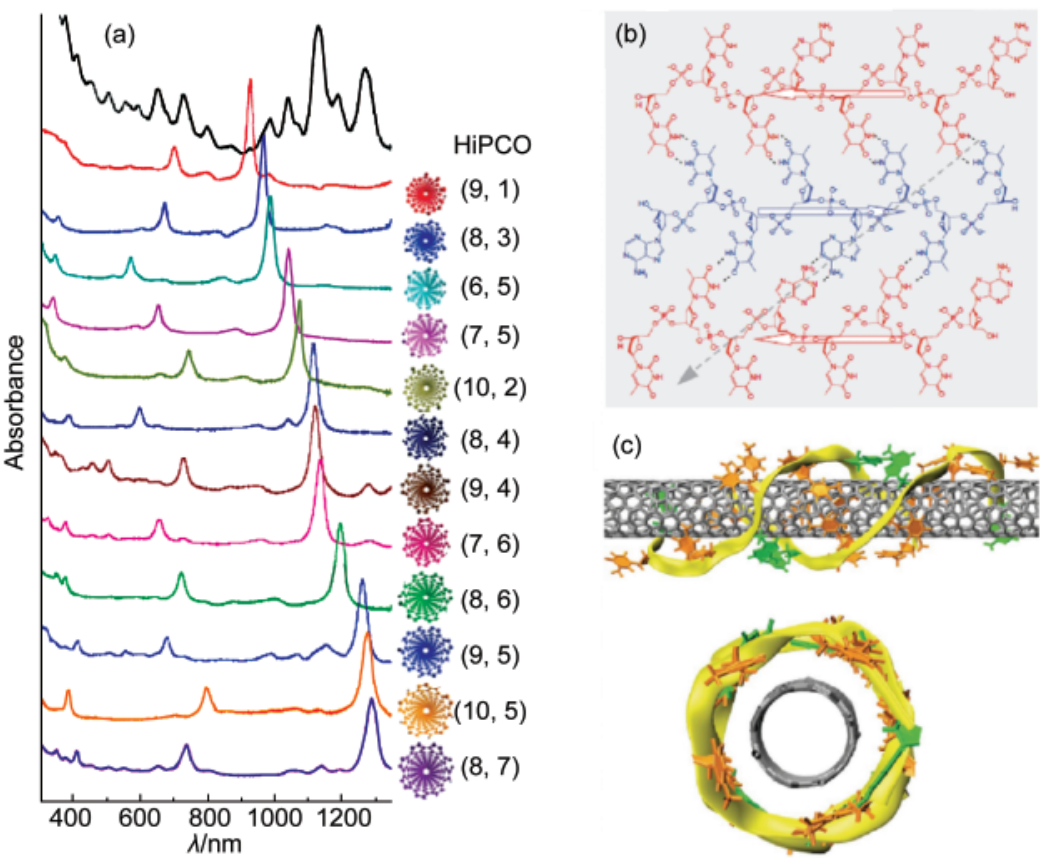

图 3 (a) 12 种分离的 s-SWCNTs 的吸收光谱及每种 SWCNTs 的截面结构及所对应的手性(沿管轴方向); (b) 由三组平行 ATTTATTT 组装的二维(2D) DNA 薄层结构以及(c) 在 $(8,4)$ 碳管表面由二维组装的 DNA 薄层缠绕形成的桶状结构 48

Fig.3 (a) Absorption spectra of 12 kinds of separated s-SWCNTs and chirality corresponding to the structure of each SWCNT species (viewed along the tube axis) and $(n, m)$ notation; (b) a two-dimensional (2D) DNA sheet structure formed by three anti-parallel ATTTATTT strands and $(c)$ a DNA barrel on a $(8,4)$ nanotube formed by rolling up a 2D DNA sheet ${ }^{48}$ HiPCO: high-pressure CO conversion

$3 \mathrm{~nm}$ 之间, 分散长度在 50 纳米至几微米, 尤其是 SWCNTs 被表面活性剂修饰荷电后, 在溶液中则与 聚电解质或 DNA 分子的性质非常相似, SWCNTs 间 相互排斥, 并且可以自由移动. 基于这些相似性, 都 促使研究者们开发基于凝胶电泳及凝胶柱色谱分 离技术下 SWCNTs 的分离.

\subsection{1 琼脂糖凝胶电泳法}

2008 年 Tanaka 研究组 ${ }^{49}$ 首先使用琼脂糖凝胶电 泳法实现了 SWCNTs 的 $\mathrm{m} / \mathrm{s}$ 分离. 他们将几种不同 方法制备的 SWCNTs 分散在 2\% SDS 水溶液中, 然 后与琼脂糖凝胶相混合, 再与空白琼脂糖凝胶相连 接, 在一定的电场力作用下, m-SWCNTs 向正极方 向迁移, s-SWCNTs 则滞留在初始凝胶中, 其分离纯 度分别为 70\%和 $95 \%$. 此外, 凝胶离心以及冷冻干燥 等技术也可以实现 SWCNTs 的 $\mathrm{m} / \mathrm{s}$ 分离, ${ }^{50}$ 并且推断 琼脂糖凝胶可以选择性地吸附 s-SWCNTs. 在此工 作基础上, 我们研究组选用不同的琼脂糖凝胶介 质 ${ }^{51}$ 以及通过 $\mathrm{NaCl}^{52}$ 的添加调制 SDS-SWCNT 表面 性质的方法, 可以一定程度地提高 $\mathrm{m}$-和 s-SWCNTs 的富集纯度.

在琼脂糖凝胶电泳法分离 $\mathrm{m}$-和 $\mathrm{s}-\mathrm{SWCNTs}$ 时,
分散剂 SDS 和琼脂糖凝胶的结合发挥着至关重要 的作用. 2010 年我们研究组 ${ }^{53}$ 报道了可以直接探测 在凝胶电泳过程中 m-SWCNTs 及 s-SWCNTs 表面 SDS 聚集结构的方法. 证实了在电场力的作用下, s-SWCNTs 表面吸附的 SDS 胶束发生脱附和迁移的 事实, 并且阐述了相比于 m-SWCNTs, s-SWCNTs 表 面具有相对低的 SDS 堆积密度. 2011 年 Tanaka 研 究组 ${ }^{54}$ 通过研究了 100 余种用表面活性剂分散的 SWCNTs 的 $\mathrm{m} / \mathrm{s}$ 分离, 阐述了只有当 SWCNTs 被类 似于 SDS 分子具有长的烷基侧链以及带有荷电头 基的表面活性剂分子包裹, 其适度的分散性使其在 m-SWCNTs 和 s-SWCNTs 表面组装成不同的结构, 从而表现出与琼脂糖凝胶间不同的亲和力. Ziegler 研究组 ${ }^{55}$ 进一步提出在 $\mathrm{m}-\mathrm{SWCNTs}$ 表面上 SDS 分子 的堆积密度相对较高, 具有取向排列结构, 表现出 与琼脂糖凝胶的亲和力较弱, 而在 s-SWCNTs 表面 上 SDS 堆积密度很低, 并且平躺在碳管表面上, 表 现出与凝胶间较强的吸附力.

\subsection{2 凝胶柱色谱技术}

利用 SDS 分散的 s-SWCNTs 在糖基凝胶中的 选择性吸附, 2009 年 Tanaka 研究组 ${ }^{56,57}$ 开展了凝胶 
柱色谱技术分离 SWCNTs 的研究工作. 他们选用琼 脂糖凝胶为分离介质, 通过 SDS 和 DOC 表面活性剂 两步淋洗程序分别获得了富集 m- 和 s-SWCNTs 溶 液, 如图 4(a)所示. 同时, Hennrich 和 Kappes 研究组 ${ }^{58}$ 使用较小孔径的氨基交联葡聚糖凝胶(Sephacryl S200)利用柱色谱技术也实现了 SWCNTs 的 $\mathrm{m} / \mathrm{s}$ 分 离. 与琼脂糖凝胶电泳相比, 所富集的 m-SWCNTs 和 s-SWCNTs 纯度有很大的提高, 并且分离过程更 简单、高效.

进一步调制分离程序和淋洗过程, 利用凝胶柱 色谱法也可以实现 SWCNTs 的直径及手性分离. 2010 年, Kataura 研究组 ${ }^{59}$ 选用市售交联琼脂糖凝胶 (Sepharose 2B), 利用多步淋洗的方法, 获得了 m-SWCNTs 以及不同直径分布的 s-SWCNTs 溶液的 富集. 随后, 利用不同手性 s-SWCNTs 与凝胶间结合 力的不同, 他们采用过载和多柱串联的方式实现了 多种手性 s-SWCNTs 的富集. ${ }^{60}$ 分离的主要思路是在 凝胶柱中加入过量的 4\% SDS 分散的 SWCNTs 溶 液, 依次通过串联的凝胶柱, 使得 s-SWCNTs 按照与
凝胶之间作用力从强到弱的顺序被依次吸附在不 同的凝胶柱中, 逐一洗脱, 如图 4(b) 所示. s-SWCNTs 的淋洗次序即吸附强弱与碳管手性结构(曲率半径) 之间的关系, 如图 4(c)所示. 他们的研究工作给出了 极具参考意义和价值的规律, 为实现更好的手性分 离提供了有意义的指导.

我们研究组 ${ }^{61}$ 发现选用更小孔尺寸的含氨基的 交联葡聚糖凝胶(Sephacryl S100) 有利于获得高纯 度的 m-SWCNTs 和 s-SWCNTs, 其分离纯度均可达 到 $95 \%$. 更为重要的是, 区别于过载及多柱串联策 略, 我们采用 SDS/DOC 的二元表面活性剂梯度淋 洗方法, 利用不同结构 s-SWCNTs 和凝胶之间亲和 力的差异, 逐渐增大淋洗液的洗脱强度也可以实 现不同手性的 s-SWCNTs 逐一洗脱, 从而实现 s-SWCNTs 的手性分离. 此外, 最近我们 ${ }^{62}$ 发现有效 的热处理和酸化处理有利于 SDS 在 m-SWCNTs 表 面上的差异化组装, 利用凝胶柱色谱法可以获得了 不同直径分布的 m-SWCNTs 溶液. 相比于其它分离 技术, 我们认为凝胶柱色谱技术与传统色谱技术相

(a)

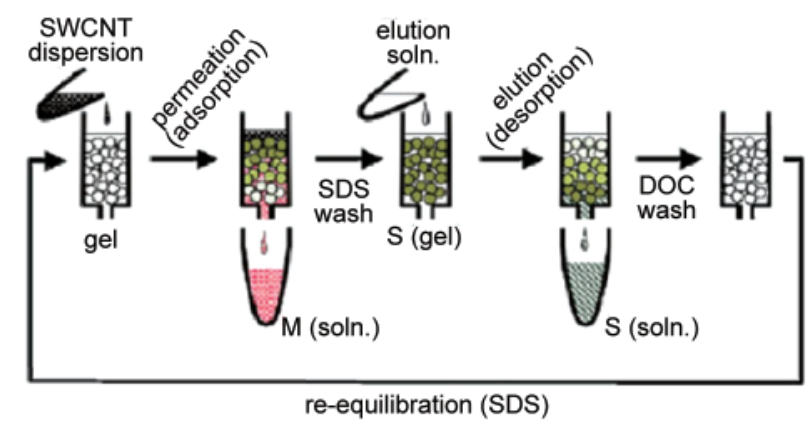

(b)

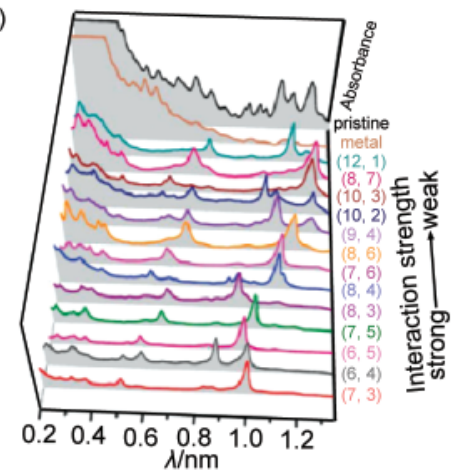

(c)

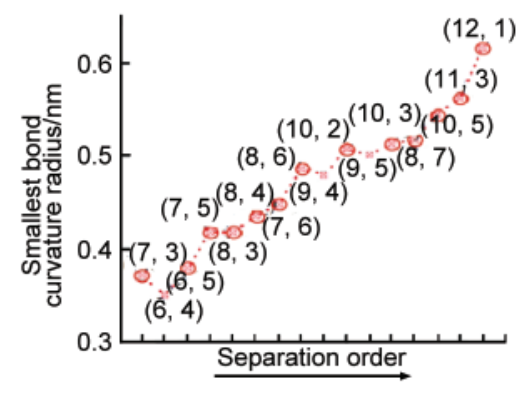

图 4 (a)凝胶柱色谱进行 m-SWCNTs (M) 和 s-SWCNTs (S) 分离过程的示意图; ${ }^{56}$ (b) 多柱凝胶柱色谱技术分离的 13 种 $(n, m)$ s-SWCNTs 以及 m-SWCNTs 和混合 HiPCO SWCNTs 的吸收光谱; ${ }^{60}(\mathrm{c})(n, m) \mathrm{s}-\mathrm{SWCNTs}$ 的分离次序与所对应的 最小 $\mathbf{C}-\mathbf{C}$ 键曲率半径间的关系 ${ }^{60}$

Fig.4 (a) Schematic of the separation progress of m-SWCNTs (M) and s-SWCNTs (S) using gel column chromatography ${ }^{56}$ (b) Absorption spectra of 13 kinds of $(n, m)$ semiconducting species together with those of metallic and pristine HiPco mixtures; ${ }^{60}$ (c) the relationship between the separation order of $(n, m)$ semiconducting species and their smallest $\mathbf{C}-\mathbf{C}$ bond curvature radius ${ }^{60}$ 
兼容, 集简单、高效、低成本、高纯度、可规模化等优 点于一身, 为最终单壁碳纳米管的规模化分离和工 业应用奠定了坚实的材料基础.

\section{6 其它方法}

除了前面介绍的常见分离方法, 其它方法也可 以实现 SWCNTs 的 $\mathrm{m} / \mathrm{s}$ 分离. 2008 年 Bao 研究组 ${ }^{63}$ 报 道了将 $\mathrm{Si} / \mathrm{SiO}_{2}$ 表面分别修饰上富含胺基和苯基硅 烷, 利用 SWCNTs 溶液旋涂的方法, 可以在基底上 直接获得 s-SWCNTs 和 m-SWCNTs 的薄膜. 同时, SWCNTs 薄膜也展现了很好的取向排列结构, 所制 备的 FET 器件具有高的开关比 $\left(9 \times 10^{5}\right)$. 在此工作基 础上, Zhang 研究组 ${ }^{64}$ 在聚二甲基硅烷(PDMS)基底 表面上分别修饰上了胺基和苯基硅烷, 通过简单的 剥离, 实现了 SWCNTs 水平阵列的 $\mathrm{m} / \mathrm{s}$ 分离. 此法克 服了基于选择性破坏技术如气相刻蚀、紫外或其它 光刻蚀等技术对 SWCNTs 结构的损伤, 也避免了基 于湿法后处理分离技术对 SWCNTs 的剪短等缺点. 尽管所获得的 s-SWCNTs 的纯度还亟待提高, 但是 由于 SWCNTs 很好地保持了原有水平取向排列和 碳管的长度, 可以方便大面积制备单一导电属性 SWCNTs 水平阵列, 从而在光电器件领域极具应用 前景. 此外, 利用含胺基化合物对半导体型碳纳米 管的选择性相互作用, 最近, Yoo 研究组 ${ }^{65}$ 使用三胺 基聚山梨醇酯 80 阳离子聚合物(TP80)将磁性纳米 粒子(MNPs)进行了化学修饰, MNPs-TP80可以选择 性地吸附在 s-SWCNTs 上, 从而在磁场的作用下, 也 实现 95\%纯度的 s-SWCNTs 的富集. 这些方法的补 充说明充分利用 SWCNTs 结构及性质差异, 开发新 的 SWCNTs 分离技术还存在很大的发展空间.

\section{3 结语与展望}

综上所述, SWCNTs 具有不同的直径、导电属性 及手性结构. 采取生长后处理的方法对其进行适当 的表面反应或化学修饰, 不同结构的 SWCNTs 会体 现出一些宏观性质的差异, 如溶解性、密度、反应活 性以及吸附力等. 这些差异使得研究者可以通过常 规分离方法, 如选择性化学修饰、交流介电泳、梯度 密度离心法、DNA包裹的离子交换色谱法、凝胶电 泳和柱色谱法等方法, 实现不同导电属性或手性结 构的 SWCNTs 分离. 进一步地, 单一导电属性或手 性结构 SWCNTs 的获得为其在高性能薄膜器件及 纳米电子器件如透明导电薄膜、FETs、光伏、传感等 领域的应用奠定了材料基础.
尽管基于选择性分离、梯度密度离心、DNA 包裹的离子交换色谱法等分离技术已经实现了 SWCNTs 的 $\mathrm{m} / \mathrm{s}$ 以及手性分离, 但是这几种分离技 术均存在效率低、成本高、难以规模化等诸多问题. 相比于上述方法, 凝胶柱色谱分离技术与传统色谱 技术相兼容, 具有简单、高效、低成本、高纯度、可规 模化等优点, 在 SWCNTs 的规模化分离和工业应用 方面存在明显的优势. 因此, 如何进一步地利用此 技术来实现不同导电属性或手性 SWCNTs 的宏量 分离将是一个重要的研究方向. 结合前期的研究 成果, 我们认为宏量分离主要面临以下问题: (1) SWCNTs 分散液的宏量制备. 现有的分散技术都还 无法实现高分散度 SWCNTs 分散液的宏量制备, 以 及超声分散带来的 SWCNTs 剪短等问题, 亟需发 展新的分散方法, 为实现高质量的 SWCNTs 宏量 分离奠定基础; (2) 宏量分离条件下, 此技术存在 SWCNTs 的 $\mathrm{m} / \mathrm{s}$ 分离纯度不高、难以精细分离等缺 点, 需要对分散剂及分离介质的结构以及分离参 数进行更细致的研究及优化; (3) 此法目前还局限 于较小管径的 SWCNTs 的分离, 对于大直径的 SWCNTs, 尤其是不同手性的 m-SWCNTs 的分离依 然是一个极大的挑战.

总之, 对 SWCNTs 分离技术的研究还仅仅是一 个开端, 未来需要建立更大规模、更低成本的分离 技术, 才能真正地推动碳纳米管在功能纳米电子器 件中的广泛应用.

\section{References}

(1) Zhang, D. H.; Ryu, K.; Liu, X. L.; Polikarpov, E.; Ly, J.; Tompson, M. E.; Zhou, C. W. Nano Lett. 2006, 6, 1880. doi: $10.1021 / \mathrm{n} 10608543$

(2) Blackburn, J. L.; Barnes, T. M.; Beard, M. C.; Kim, Y. H.; Tenent, R. C.; McDonald, T. J.; To, B.; Coutts, T. J.; Heben, M. J. ACS Nano 2008, 2, 1266. doi: 10.1021/nn800200d

(3) Durkop, T.; Getty, S. A.; Cobas, E.; Fuhrer, M. S. Nano Lett. 2004, 4, 35. doi: 10.1021/n1034841q

(4) Kong, J.; Franklin, N. R.; Zhou, C. W.; Chapline, M. G.; Peng, S.; Cho, K. J.; Dai, H. J. Science 2000, 287, 622. doi: 10.1126/ science.287.5453.622

(5) Javey, A.; Guo, J.; Wang, Q.; Lundstrom, M.; Dai, H. J. Nature 2003, 424, 654. doi: 10.1038/nature01797

(6) Wind, S. J.; Appenzeller, J.; Avouris, P. Phys. Rev. Lett. 2003, 91, 058301. doi: 10.1103/PhysRevLett.91.058301

(7) Li, Y. M.; Mann, D.; Rolandi, M.; Kim, W.; Ural, A.; Hung, S.; Javey, A.; Cao, J.; Wang, D. W.; Yenilmez, E.; Wang, Q.; Gibbons, J. F.; Nishi, Y.; Dai, H. J. Nano Lett. 2004, 4, 317. doi: 
$10.1021 / \mathrm{n} 1035097 \mathrm{c}$

(8) Qu, L. T.; Du, F.; Dai, L. M. Nano Lett. 2008, 8, 2682. doi: $10.1021 / \mathrm{n} 1800967 \mathrm{n}$

(9) Hong, G.; Zhang, B.; Peng, B. H.; Zhang, J.; Choi, W. M.; Choi, J. Y.; Kim, J. M.; Liu, Z. F. J. Am. Chem. Soc. 2009, 131, 14642. doi: $10.1021 /$ ja 9068529

(10) Ding, L.; Tselev, A.; Wang, J.; Yuan, D.; Chu, H.; McNicholas, T. P.; Li, Y.; Liu, J. Nano Lett. 2009, 9, 800. doi: 10.1021/ n1803496s

(11) Xu, Z.; Lu, W. G.; Wang, W. L.; Gu, C. Z.; Liu, K. H.; Bai, X. D.; Wang, E. G.; Dai, H. J. Adv. Mater. 2008, 20, 3615. doi: 10.1002/adma.v20:19

(12) Yao, Y. G.; Feng, C. Q.; Zhang, J.; Liu, Z. F. Nano Lett. 2009, 9, 1673. doi: $10.1021 / \mathrm{n} 1900207 \mathrm{v}$

(13) Yu, X. C.; Zhang, J.; Choi, W.; Choi, J. Y.; Kim, J. M.; Gan, L. B.; Liu, Z. F. Nano Lett. 2010, 10, 3343. doi: 10.1021/nl1010178

(14) Collins, P. C.; Arnold, M. S.; Avouris, P. Science 2001, 292, 706. doi: 10.1126/science. 1058782

(15) Hassanien, A.; Tokumoto, M.; Umek, P.; Vrbanic, D.; Mozetic, M.; Mihailovic, D.; Venturini, P.; Pejovnik, S. Nanotechnology 2005, 16, 278. doi: 10.1088/0957-4484/16/2/017

(16) Zhang, Y. Y.; Zhang, Y.; Xian, X. J.; Zhang, J.; Liu, Z. F. Journal of Physical Chemistry C 2008, 112, 3849. doi: 10.1021/ jp710691j

(17) Huang, H. J.; Maruyama, R.; Noda, K.; Kajiura, H.; Kadono, K. J. Phys. Chem. B 2006, 110, 7316.

(18) Zhang, H. L.; Liu, Y. Q.; Cao, L. C.; Wei, D. C.; Wang, Y.; Kajiura, H.; Li, Y. M.; Noda, K.; Luo, G. F.; Wang, L.; Zhou, J.; Lu, J.; Gao, Z. X. Adv. Mater. 2009, 21, 813. doi: 10.1002/adma. v21:7

(19) Strano, M. S.; Dyke, C. A.; Usrey, M. L.; Barone, P. W.; Allen, M. J.; Shan, H. W.; Kittrell, C.; Hauge, R. H.; Tour, J. M.; Smalley, R. E. Science 2003, 301, 1519. doi: 10.1126/ science. 1087691

(20) Doyle, C. D.; Rocha, J. D. R.; Weisman, R. B.; Tour, J. M. J. Am. Chem. Soc. 2008, 130, 6795. doi: 10.1021/ja800198t

(21) An, K. H.; Park, J. S.; Yang, C. M.; Jeong, S. Y.; Lim, S. C.; Kang, C.; Son, J. H.; Jeong, M. S.; Lee, Y. H. J. Am. Chem. Soc. 2005, 127, 5196. doi: 10.1021/ja0428199

(22) Banerjee, S.; Wong, S. S. J. Am. Chem. Soc. 2004, 126, 2073. doi: 10.1021/ja038111w

(23) Menard-Moyon, C.; Izard, N.; Doris, E.; Mioskowski, C. J. Am. Chem. Soc. 2006, 128, 6552. doi: 10.1021/ja060802f

(24) Kamaras, K.; Itkis, M. E.; Hu, H.; Zhao, B.; Haddon, R. C. Science 2003, 301, 1501. doi: 10.1126/science.1088083

(25) Chattopadhyay, D.; Galeska, L.; Papadimitrakopoulos, F. J. Am. Chem. Soc. 2003, 125, 3370. doi: 10.1021/ja0285991

(26) Chen, Z. H.; Du, X.; Du, M. H.; Rancken, C. D.; Cheng, H. P.; Rinzler, A. G. Nano Lett. 2003, 3, 1245. doi: 10.1021/n10344763

(27) Li, H. P.; Zhou, B.; Lin, Y.; Gu, L. R.; Wang, W.; Fernando, K. A. S.; Kumar, S.; Allard, L. F.; Sun, Y. P. J. Am. Chem. Soc.
2004, 126, 1014. doi: $10.1021 /$ ja037142o

(28) Ju, S. Y.; Doll, J.; Sharma, I.; Papadimitrakopoulos, F. Nature Nanotechnology 2008, 3, 356. doi: 10.1038/nnano.2008.148

(29) Wang, F.; Matsuda, K.; Rahman, A. F. M. M.; Peng, X. B.; Kimura, T.; Komatsu, N. J. Am. Chem. Soc. 2010, 132, 10876. doi: 10.1021/ja1044677

(30) Nish, A.; Hwang, J. Y.; Doig, J.; Nicholas, R. J. Nature Nanotechnology 2007, 2, 640.

(31) Lee, H. W.; Yoon, Y.; Park, S.; Oh, J. H.; Hong, S.; Liyanage, L. S.; Wang, H. L.; Morishita, S.; Patil, N.; Park, Y. J.; Park, J. J.; Spakowitz, A.; Galli, G.; Gygi, F.; Wong, P. H. S.; Tok, J. B. H.; Kim, J. M.; Bao, Z. A. Nature Communications 2011, 2, 541. doi: $10.1038 /$ ncomms 1545

(32) Wang, W. Z.; Li, W. F.; Pan, X. Y.; Li, C. M.; Li, L. J.; Mu, Y. G.; Rogers, J. A.; Chan-Park, M. B. Adv. Funct. Mater. 2011, 21, 1643. doi: $10.1002 /$ adfm. 201002278

(33) Gao, J.; Kwak, M.; Wildeman, J.; Hermann, A.; Loi, M. A. Carbon 2011, 49, 333. doi: 10.1016/j.carbon.2010.09.036

(34) Lemasson, F.; Berton, N.; Tittmann, J.; Hennrich, F.; Kappes, M. M.; Mayor, M. Macromolecules 2012, 45, 713. doi: 10.1021/ ma201890g

(35) Imin, P.; Imit, M.; Adronov, A. Macromolecules 2011, 44, 9138. doi: 10.1021/ma201610y

(36) Krupke, R.; Hennrich, F.; von Lohneysen, H.; Kappes, M. M. Science 2003, 301, 344. doi: 10.1126/science.1086534

(37) Shin, D. H.; Kim, J. E.; Shim, H. C.; Song, J. W.; Yoon, J. H.; Kim, J.; Jeong, S.; Kang, J.; Baik, S.; Han, C. S. Nano Lett. 2008, 8, 4380. doi: 10.1021/n1802237m

(38) Arnold, M. S.; Stupp, S. I.; Hersam, M. C. Nano Lett. 2005, 5, 713. doi: $10.1021 / \mathrm{n} 1050133 \mathrm{o}$

(39) Arnold, M. S.; Green, A. A.; Hulvat, J. F.; Stupp, S. I.; Hersam, M. C. Nature Nanotechnology 2006, 1, 60. doi: 10.1038/ nnano.2006.52

(40) Antaris, A. L.; Seo, J. W. T.; Green, A. A.; Hersam, M. C. ACS Nano 2010, 4, 4725. doi: 10.1021/nn101363m

(41) Green, A. A.; Duch, M. C.; Hersam, M. C. Nano Research 2009, 2, 69. doi: 10.1007/s12274-009-9006-y

(42) Zhao, P.; Einarsson, E.; Xiang, R.; Murakami, Y.; Maruyama, S. Journal of Physical Chemistry C 2010, 114, 4831.

(43) Ghosh, S.; Bachilo, S. M.; Weisman, R. B. Nature Nanotechnology 2010, 5, 443. doi: 10.1038/nnano.2010.68

(44) Zheng, M.; Jagota, A.; Semke, E. D.; Diner, B. A.; Mclean, R. S.; Lustig, S. R.; Richardson, R. E.; Tassi, N. G. Nature Materials 2003, 2, 338. doi: 10.1038/nmat877

(45) Zheng, M.; Jagota, A.; Strano, M. S.; Santos, A. P.; Barone, P.; Chou, S. G.; Diner, B. A.; Dresselhaus, M. S.; McLean, R. S.; Onoa, G. B.; Samsonidze, G. G.; Semke, E. D.; Usrey, M.; Walls, D. J. Science 2003, 302, 1545. doi: 10.1126/ science.1091911

(46) Zheng, M.; Semke, E. D. J. Am. Chem. Soc. 2007, 129, 6084. doi: $10.1021 / \mathrm{ja} 071577 \mathrm{k}$ 
(47) Zhang, L.; Zaric, S.; Tu, X. M.; Wang, X. R.; Zhao, W.; Dai, H. J. J. Am. Chem. Soc. 2008, 130, 2686. doi: 10.1021/ja7106492

(48) Tu, X. M.; Manohar, S.; Jagota, A.; Zheng, M. Nature 2009, 460, 250. doi: $10.1038 /$ nature 08116

(49) Tanaka, T.; Jin, H. H.; Miyata, Y.; Kataura, H. Applied Physics Express 2008, 1, 114001. doi: 10.1143/APEX.1.114001

(50) Tanaka, T.; Jin, H.; Miyata, Y.; Fujii, S.; Suga, H.; Naitoh, Y.; Minari, T.; Miyadera, T.; Tsukagoshi, K.; Kataura, H. Nano Lett. 2009, 9, 1497. doi: 10.1021/n18034866

(51) Zhang, J.; Wen, X. N.; Li, H. B.; Jin, H. H.; Song, Q. J.; Li, Q. W. Chemical Journal of Chinese Universities 2010, 31, 2190. [张 静, 温晓南, 李红波, 金赫华, 宋启军, 李清文. 高等学校 化学学报, 2010, 31, 2190.]

(52) Wen, X. N.; Zhang, J.; Gu, W. X.; Jin, H. H.; Li, H. B.; Li, Q. W. Acta Physico-Chimica Sinica 2010, 26, 2757. [温晓南, 张 静, 顾文秀, 金赫华, 李红波, 李清文. 物理化学学报, 2010, 26, 2757.] doi: 10.3866/PKU.WHXB20100932

(53) Li, H. B.; Jin, H. H.; Zhang, J.; Wen, X. N.; Song, Q. J.; Li, Q. W. Journal of Physical Chemistry C 2010, 114, 19234. doi: 10.1021/jp106869r

(54) Tanaka, T.; Urabe, Y.; Nishide, D.; Kataura, H. J. Am. Chem. Soc. 2011, 133, 17610. doi: 10.1021/ja208221g

(55) Silvera-Batista, C. A.; Scott, D. C.; McLeod, S. M.; Ziegler, K. J. Journal of Physical Chemistry C 2011, 115, 9361. doi: 10.1021/jp111349x

(56) Tanaka, T.; Urabe, Y.; Nishide, D.; Kataura, H. Applied Physics
Express 2009, 2, 125002. doi: 10.1143/APEX.2.125002

(57) Tanaka, T.; Urabe, Y.; Nishide, D.; Liu, H. P.; Asano, S.; Nishiyama, S.; Kataura, H. Physica Status Solidi B-Basic Solid State Physics 2010, 247, 2867. doi: 10.1002/pssb.v247.11/12

(58) Moshammer, K.; Hennrich, F.; Kappes, M. M. Nano Research 2009, 2, 599. doi: 10.1007/s12274-009-9057-0

(59) Liu, H.; Feng, Y.; Tanaka, T.; Urabe, Y.; Kataura, H. Journal of Physical Chemistry C 2010, 114, 9270.

(60) Liu, H. P.; Nishide, D.; Tanaka, T.; Kataura, H. Nature Communications 2011, 2, 309. doi: 10.1038/ncomms1313

(61) Gui, H.; Li, H. B.; Tan, F. R.; Jin, H. H.; Zhang, J.; Li, Q. W. Carbon 2012, 50, 332. doi: 10.1016/j.carbon.2011.08.034

(62) Zhang, J.; Tan, F.; Li, H.; Jin, H.; Li, Q. Physica Status Solidi (RRL) - Rapid Research Letters 2012, 6, 250. doi: 10.1002/pssr. v6.6

(63) LeMieux, M. C.; Roberts, M.; Barman, S.; Jin, Y. W.; Kim, J. M.; Bao, Z. N. Science 2008, 321, 101. doi: 10.1126/ science. 1156588

(64) Hong, G.; Zhou, M.; Zhang, R. O. X.; Hou, S. M.; Choi, W.; Woo, Y. S.; Choi, J. Y.; Liu, Z. F.; Zhang, J. Angewandte Chemie-International Edition 2011, 50, 6819. doi: 10.1002/ anie. 201101700

(65) Kim, H. J.; Hwang, S.; Oh, J.; Chang, Y. W.; Lim, E. K.; Haam, S.; Kim, C. S.; Yoo, K. H. Nanotechnology 2011, 22, 045703. doi: 10.108810957-4484122141045703 